\title{
Integrating DPWS and OPC UA device-level SOA features into IEC 61850 applications
}

\author{
Stjepan Sučić \\ Končar-KET, Zagreb, Croatia \\ stjepan.sucic@koncar-ket.hr
}

\author{
Bernard Bony, Laurent Guise, François Jammes \\ Schneider Electric, Grenoble, France \\ \{bernard.bony, laurent.guise, francois.jammes2\} \\ @schneider-electric.com
}

\author{
Ante Marušić \\ University of Zagreb, Zagreb, Croatia \\ ante.marusic@fer.hr
}

\begin{abstract}
Flexible and adaptable integration of diverse devices and applications used for power system automation is one of the key prerequisites for accelerated and future-proof Smart Grid deployment. International standard IEC 61850 has been globally accepted as one of the prime movers of successful and interoperable Smart Grid integration. However, current IEC 61850 definition lacks the integration capability based on device-level Service Oriented Architecture (SOA) paradigm. SOA is widely applied for enterprise integration and one of the envisioned paradigms for industrial automation domain. Implementing IEC 61850 systems according to device-level SOA principles could significantly facilitate standards-compliant device integration and accelerate overall Smart Grid automation deployment.
\end{abstract}

This article presents a solution for integrating device-level SOA principles into IEC 61850 architecture. Two most prominent device-level SOA technologies, Devices Profile for Web Services (DPWS) and OPC Unified Architecture (OPC UA), have been analyzed according to IEC 61850 system requirements. As a result of analysis, a novel middleware platform which integrates both DPWS and OPC UA features into IEC 61850 applications is presented. The proposed solution provides an extensible Smart Grid application framework based on state-of-the-art industrial automation standards thus allowing accelerated Smart Grid integration.

\section{INTRODUCTION}

The electrical power grids of the future, also known as the Smart Grids [1], introduce numerous challenges and novelties to all elements of traditional power systems. The indispensable Smart Grid goal, two-way flow of power and information, is hampered by usage of non-interoperable and proprietary automation solutions. Hence, one of the key prerequisites of successful Smart Grid deployment is streamlined and seamless standards-compliant automation integration [1]. The international standard IEC 61850 [2] is foreseen as a solution which provides extensible Smart Grid integration architecture based on vendor-independent and technology-neutral principles. Nevertheless the standard was initially designed mainly for substation automation and not for novel Smart Grid assets such as virtual power plants (VPPs) [3] and electric vehicle supply stations [4]. Therefore, there are undergoing standardization efforts with aim to find suitable technical solution that will complement current IEC 61850 definition in order to create future-proof and adaptable Smart grid integration framework. One possible solution for surpassing aforementioned issues is integration of device-level Service Oriented Architecture
(SOA) [5] design principles into IEC 61850 applications. SOA is a well accepted application integration principle in enterprise domain [6] and one of the envisioned integration paradigms for the real-time industrial automation [7].

This paper provides an analysis of IEC 61850 application architecture requirements regarding SOA-based integration and presents capabilities of a middleware platform based on Web Services. Therefore, two most influential device-level SOA technologies, Devices Profile for Web Services (DPWS) [8] and OPC Unified Architecture (OPC UA) [9], have been evaluated. As a result of analysis, a novel middleware approach which integrates both DPWS and OPC UA features into IEC 61850 applications, is proposed. The presented solution provides a service-oriented automation middleware framework which is compliant to industrial standards and can significantly contribute to accelerated integration of Smart Grid devices and applications by utilizing device-level SOA principles.

The rest of the paper is organized as follows. The next section provides an overview of relevant related work while the section III gives a short introduction to IEC 61850 standard. The section IV identifies IEC 61850 application design issues resulting from Smart Grid integration requirements and introduces DPWS and OPC UA as a potential solution approaches. Finally, the coexistence of device-level SOA technologies in proposed middleware architecture for IEC 61850 systems is presented in section V.

\section{SERVICE-ORIENTED SMART GRID AUTOMATION - STATE OF THE ART}

\section{A. Smart Grid automation vision}

Smart Grid involves significant number of heterogeneous and geographically dispersed actors with intermittent energy sources such as distributed energy resources (DERs), energy storage systems and electric vehicles (EVs) [4], [10]. Integration of these Smart Grid entities has resulted in novel system management architectures such as VPPs [3] and smart homes/buildings [11] while also introducing new solutions for optimized control [10]. Fulfilling Smart Grid vision, i.e. enabling two-way flow of power and information, and creating prosumers (power grid actors which are both producers and consumers of electricity) has several prerequisites in a form of communication [12] and electrical infrastructure [13] along with intelligent automation systems 
[14]. Streamlined and standards-compliant integration is indispensable requirement for successful and secure Smart Grid automation [1][15].

IEC 61850 provides standardized communication interface for controlled entities in technology-neutral manner while separating data semantics from the data exchange services [16]. Therefore, IEC 61850 definitions can be easily extended to overall Smart Grid domain. Today, IEC 61850 is one of the most influential industrial automation standards [1] and has been recognized as a promising candidate that could fulfill identified Smart Grid automation gaps as well as provide an adaptable integration framework solution.

\section{B. Service-oriented Automation}

For years industrial automation was based on specialized and single-purpose systems. Paradigm shift from centralized and tightly-coupled systems towards decentralized and adaptable automation architectures [14] is accompanied with new software integration techniques and modular applications. One of the most prominent design paradigms for next-generation automation applications is based on SOA principles as shown in [5] and [7]. SOA is based on system decomposition into a set of services which reflect process functions. Services are defined as autonomous, platformindependent entities which can be described, published, discovered and assembled [17]. SOA paradigm is considered as an effective approach for building integrated, heterogeneous and distributed applications.

Introducing SOA paradigm into industrial automation systems has brought capability of reusing existing solutions, expanding application domain and creating generic automation service components [18]. Also, one of the outcomes of SOA-based automation is development of embedded, device-level SOA technologies [5]. Two most influential device-level SOA technologies which are results of industrial consortium research projects and standardization bodies are DPWS [8] and OPC UA [9]. Despite having competitive technical approaches their cooperation is foreseen as a feasible solution providing significant valueadded features for service-oriented industrial automation as shown in [19], [20], [21] and [22].

\section{Device-level SOA for the Smart Grid}

Although IEC 61850 is a globally accepted automation approach, its current definition and integration aspects are inadequate to compete with dynamic and reconfigurable Smart Grid topology [23]. This problem can be solved by

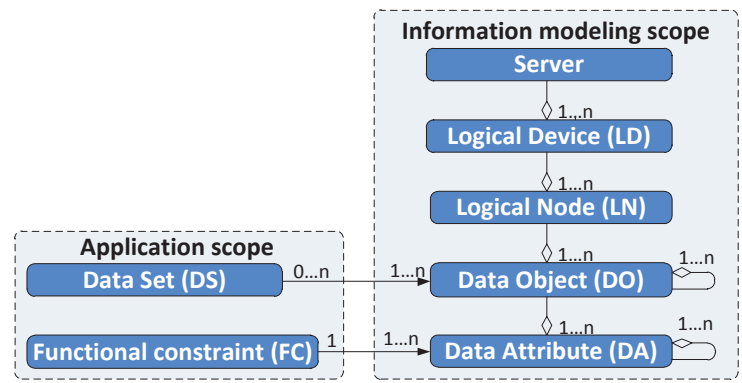

Fig. 1. IEC 61850 data class model [24]. introducing SOA design principles into IEC 61850 application architecture as shown in [3]. There have been several approaches for complementing IEC 61850 with SOA capability based on OPC UA [23] and DPWS [24]. However, solution presented in this paper integrates both DPWS and OPC UA approaches with IEC 61850 in order to create unified device-level SOA middleware for the Smart Grid.

\section{IEC 61850 - SMART GRID AUTOMATION ARCHITECTURE}

Although often seen as a yet another telecontrol protocol, IEC 61850 is considerably more than a set of encoding rules and data retrieval mechanisms for remote control of field devices. IEC 61850 [2] is more appropriately described as an automation architecture which defines requirements for vertical and horizontal communication services along with standardized data semantics. The following chapters provide an outline of the main IEC 61850 features.

\section{A. Semantic-enabled Smart Grid}

One of the essential novelties introduced by IEC 61850 , compared to other approaches, is standardized data semantics used for subsystem automation functions. IEC 61850 semantic data description is optimized for centralized and hierarchically organized supervisory control applications. Data model structure is based on object-oriented design of information relevant for process automation [16].

Relationships between IEC 61850 data model classes are shown in Fig.1. Detailed description about IEC 61850 semantic modeling principles is out of scope of this paper and can be found in [16], [23] and [24].

IEC 61850 modeling concepts are easily applicable to a number of Smart Grid entities. Already existing IEC 61850 definitions [2] cover substations, hydro power plants, DERs and inverters. It is expected that in near future these definitions will also be expanded for energy storage systems, EV supply stations, smart homes/buildings and many other industrial automation interfaces.

\section{B. Data-exchange models}

Communication between IEC 61850 applications and devices is based on technology-neutral specifications known as Abstract Communication Service Interfaces (ACSI). ACSI are formed as a set of rules and state machines defining data exchange procedures and mechanisms [16]. They include definitions and interface requirements for peer-to-peer communication aspects, i.e. transferring digitalized measurements (Sampled Measured Values - SMV) and protective interlocking logic (Generic Object Oriented Substation Event - GOOSE), as well as vertical communication services required for application-level integration. IEC 61850 application design aspects described in this paper are dealing only with vertical communication services and thus, only these services are discussed further.

\section{IEC 61850 implementation issues}

Implementation of IEC 61850 vertical communication services can be done by a variety of general-purpose middleware technologies. Currently, only official IEC 61850 
mapping middleware is Manufacturing Message Specification (MMS) [25]. MMS-based implementations have been successfully applied for years in substation automation domain what definitively contributed to accelerated dissemination of IEC 61850 devices.

MMS [26] is application-level message-oriented middleware with binary serialized data encoding. It is optimized for low bandwidth networks and distributed soft real-time automation applications. However, MMS is rather complex, it is based on full OSI stack, it has no integrated security mechanisms and it involves long learning curve. These issues make IEC 61850 applications development unnecessarily difficult and over-engineered.

For the above identified issues, IEC's Technical Committee 57 (TC57) has started new standardization efforts in order to find suitable technical solution that will complement IEC 61850 standard for new Smart Grid use cases. Solution proposed in this paper fulfills identified Smart Grid integration gaps and it is potential choice for future IEC 61850 middleware definition based on Web Services.

\section{ANALYZING SMART GRID MidDLEWARE FOR DEVICE-LEVEL SOA APPLICATIONS}

\section{A. Novel Smart Grid application design requirements}

Large scale deployment of Smart Grid entities such as DERs, EVs, energy storage systems and smart meters have introduced new control models like VPPs, microgrids and smart houses/buildings. Aforementioned novelties have also brought a set of original application design requirements for Smart Grid automation. By analyzing available documentation [1], [2], [12], these have been identified as follows:

- Platform-independent and standard-compliant;

- Distributed applications with real-time execution;

- Embedded applications with enterprise-level integration capability;

- Event-driven service-oriented architecture;

- Semantic-enabled data exchange;

- Dynamic reconfiguration and performance scalability;

- Plug-and-play capability.

These requirements outline complexity of interactions among Smart Grid entities and establish foundations for future-proof automation application design. IEC 61850 architecture is defined in an abstract manner and hence, it is generic enough to be complemented with suitable middleware platform in order to fulfill identified requirements. As previously stated, there are several significant issues when applying MMS for developing Smart Grid applications. Therefore, integration of device-level SOA design principles into IEC 61850 application is proposed. The following chapters provide an outline of DPWS and OPC UA as a solutions for SOA-based IEC 61850 architecture.

\section{B. Devices Profile for Web Services}

DPWS [8] is a specific profile of Web service protocols enabling SOA capabilities on resource constrained devices. It is partially based on Web Services Architecture (WSA) [27] which enables creating highly-composable and incrementallyintegrated protocol stacks. The main difference from WSA is that DPWS does not require centralized service repository but it uses multicast service discovery instead.

1) DPWS as IEC 61850 middleware: A strong argument for using DPWS for Smart Grid automation is a number of available open source toolkits and significant community support resulting from industrial research projects [28], [29]. However, in order to implement IEC 61850 architecture based on DPWS protocol stack it is necessary to additionally develop customized application-level services which fulfill ACSI functional requirements. An example of possible solution is Web service mapping found in [30]. However, in order to extract semantic data from a DPWS server, a generic Web Services client has to analyze XML schema documents and resolve data structures. There is no built-in metamodeling capability in DPWS that could help in this task. For this reason, it cannot be regarded as a complete solution for an entire application-level mapping for ACSI. DPWS implements the lower-layer application mechanisms and transport profile, enabling development of customized application-level services.

\section{C. $O P C$ Unified Architecture}

OPC UA [9] is a state-of-the-art device-level SOA technology and successor of OPC as one of the most influential technologies in industrial automation integration. Contrarily to OPC, OPC UA defines a set of application-level services which are independent on implementation technology and it also has information modeling capability.

1) Main OPC UA features: OPC UA Services are based on defining request and response parameters that are conveyed between client and server. They are logically grouped into service sets that enable: server discovery, session manipulation, address space management and also pollingbased and event-driven data exchange. The services can be implemented as $U A$ Web Services and UA Native services. $U A W e b$ Services are based on set of WS-* specifications similar to DPWS as described in [20], while UA Native uses simple binary network protocol directly on top of TCP/IP.

The other important OPC UA feature is a meta-modeling mechanism based on an AddressSpace using object-oriented concepts for creating the collection of semantic data that OPC UA servers provide to the OPC UA clients.

2) OPC UA as IEC 61850 middleware: The OPC UA features described above show that OPC UA fulfills quite well the requirements for IEC 61850 architecture. ACSI concept is very similar to OPC UA Services, while AddressSpace can be used for modeling IEC 61850 data semantics. Additionally, OPC UA specifies several protocol stacks defining usage of specific implementation technologies. Nevertheless, a notable issue for the compliance with IEC 61850 is the event-driven data exchange mechanism. OPC UA enables notification-based communication by combining MonitoredItem Service Set and Subscription Service Set. Although, being designated as event-driven, these services are in fact based on periodic request/response pattern. It can be easily described as polling 
of notification queues according to subscription. Therefore, this data exchange is not really event-driven and asynchronous what is one of the crucial scalability requirements for Smart Grid applications. This is caused by selection of lower-layer WS-* specifications for OPC UA which do not support asynchronous HTTP-based data exchange. In case of DPWS, this feature is available by using WS-Eventing specification.

Despite abovementioned issues, IEC 61850 can be partially mapped to OPC UA as it is shown in [23].

\section{SERVICE-ORIENTED AUtOMATION WITH IEC 61850}

Integrating device-level SOA design principles into Smart Grid applications requires implementation technology capable to fulfill all requirements of IEC 61850 architecture. Neither DPWS nor OPC UA completely accomplishes this goal. Therefore, a combined approach which utilizes merged DPWS and OPC UA components is proposed.

\section{A. Converged device-level SOA middleware for IEC 61850}

In order to design adequate IEC 61850 middleware platform, the main benefits from DPWS and OPC UA were taken into account: extensibility of DPWS protocol stack, standard-compliant OPC UA application-level services and AddressSpace meta-modeling capability.

The converged middleware architecture is based on integrating OPC UA Services into DPWS protocol stack as Hosted services. Therefore, the problem of creating devicelevel SOA middleware for IEC 61850 is narrowed to finding specific profile of OPC UA Services which fulfills ACSI service requirements.

The architectural overview of novel device-level SOA middleware architecture for IEC 61850 applications is presented in Fig. 2. It outlines relationships between DPWS stack and OPC UA Services required for ACSI functionalities. The middleware mapping details and distinctive features are described in the following sections.

\section{B. Service alignment}

The first mapping step is finding functionally equivalent OPC UA services $\left(U A_{\text {services }}\right)$ which fulfill vertical ACSI
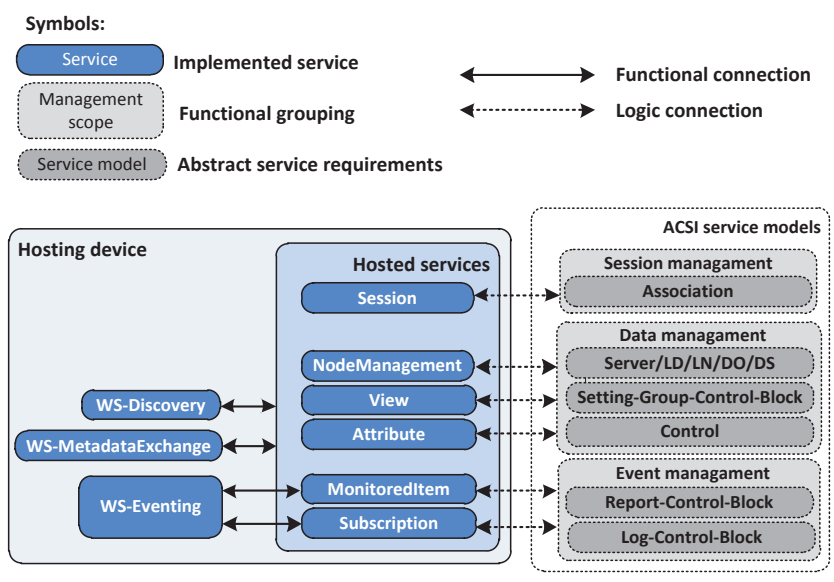

Fig. 2. Architecture of device-level SOA middleware for IEC 61850 service $\left(A C S I_{\text {vertical }}\right)$ requirements. $U A_{\text {services }}$ and $A C S I_{\text {vertical }}$ have several overlapping modeling approaches and thus require detailed service alignment analysis.

\section{1) Association control}

The first action for vertically integrated IEC 61850 applications is creation of two-party associations which enable reliable end-to-end information flow control (acsi $\left.i_{\text {asso }}\right)$. With OPC UA this can be fulfilled with help of Session Service Set ( $u a_{\text {sess }}$ ). These services provide uniquely identified application layer connection establishment and can be mapped based on one-to-one relationship with acsiasso (1).

$$
a c s i_{\text {asso }} \cong u a_{\text {sess }}
$$

\section{2) Information model management}

IEC 61850 information modeling and browsing services $\left(a c s i_{\text {inform }}\right)$ are dependable on data structure levels. However, OPC UA defines single NodeManagement Service Set $\left(u a_{n o m a}\right)$ for managing complete AddressSpace. Subsets of data model can be organized with View Service Set (uaview) while reading and writing data to endpoint attributes is done by Attribute Service Set $\left(u a_{\text {attr }}\right)$. Therefore, three aforementioned $U A_{\text {services }}$ combined fulfill IEC 61850 information modeling requirements (2). An example how to model IEC 61850 data model with OPC UA AddressSpace components is shown in [23].

$$
\text { acsi }_{\text {inform }} \subseteq\left(u a_{\text {nота }} \cup u a_{\text {view }} \cup u a_{\text {attr }}\right)
$$

\section{3) Device management}

Complex control block structures responsible for sending control commands $\left(a c s i_{c t r l}\right)$ and editing device setting groups $\left(a c s i_{s g c b}\right)$ are directly influenced by reading and writing specific data values on device-level. Therefore, these $A C S I_{\text {vertical }}$ service models can be directly mapped to $u a_{\text {attr }}$ (3).

$$
\left(a c s i_{c t r l} \cup a c s i_{s g c b}\right) \cong u a_{a t t r}
$$

\section{4) Event-driven data-exchange}

Event-driven data exchange is one of the crucial requirements for future Smart Grid applications. Integrating event-driven architecture (EDA) principles enables scalable and flexible application design and additionally, optimizes communication bandwidth usage in distributed systems [31]. In case of IEC 61850 applications, EDA design is based on using Report-Control-Block (RCB) ACSI service models $\left(a c s i_{r c b}\right)$.

IEC 61850 events are the new data attribute values resulting from changed state of field devices (e.g. breaker trips) or reached threshold for supervised measurements. One or more events, together with related information, are grouped into IEC 61850 report and sent from the device towards the report handler application. IEC 61850 specifies two $a c s i_{r c b}$ class models [16], the buffered RCB (BRCB) responsible for buffering events at device level in order to guarantee sequence-of-events in case of loss of connection, and unbuffered RCB (URCB) responsible for sending the reports on a "best effort" basis. $a c s i_{r c b}$ models involve three basic services: 
- SetRCBValues: Service used for managing settings of RCB object on server side. Used in particular to enable/disable the event-driven data exchange.

- GetRCBValues: Service used for retrieving settings of RCB object from server.

- Report: Service supporting the spontaneous data delivery mechanism.

As previously stated, OPC UA defines two applicationlevel service sets. MonitoredItem Service Set $\left(u_{\text {monit }}\right)$ allows OPC UA clients to create, modify, and delete MonitoredItems (items used to monitor Attributes for value changes). Subscription Service Set $\left(u a_{\text {subs }}\right)$ allows OPC UA clients to create, modify and delete Subscriptions (collections of MonitoredItems which send Notifications to the client). These services sets are similar to $a c s i_{r c b}$ service models, excepted for the asynchronous behavior of HTTP-based data exchange. In this paper it is proposed how to combine $u a_{\text {subs }}$ and $u a_{\text {monit }}$ services with WS-Eventing $\left(W S_{\text {event }}\right)$ specification.

Mapping IEC 61850 reports to DPWS and OPC UA: Event-driven notification delivery mechanism for DPWS platform is defined by $W S_{\text {event }}$ specification. The publish/subscribe paradigm of $W S_{\text {event }}$ involves an Event source, a Subscriber, an Event sink and a Subscription manager. $W S_{\text {event }}$ includes several subscription related operations: Subscribe, Renew, GetStatus, Unsubscribe and SubscriptionEnd. The relationship among $W S_{\text {event }}$ entities and respective operations is depicted in Fig. 3. Message notification is based on "push" as the default delivery mode which is completely asynchronous what fulfills one of the most important Smart Grid application requirements.

From a $W S_{\text {event }}$ perspective the IEC 61850 application is the Event sink. In order to be consistent with the $a c s i_{r c b}$, the Subscriber, Subscription manager and Event source are parts of the IEC 61850 device. This is possible since $W S_{\text {event }}$ allows decoupling the Event sink from the Subscriber. Subscription process, on IEC 61850 application side, starts with receiving a SetRCBValues service request. This request is mapped to adequate OPC UA service requests, i.e. consecutive call of CreateMonitoredItems and CreateSubscription services. The IEC 61850 device extracts the relevant SOAP message payload which is used for setting an RCB object. Then, the Subscriber sends a Subscribe request to the Subscription Manager/Event source.

$W S_{\text {event }}$ events are formed as OPC UA Notifications which correspond to IEC 61850 reports according to requirements of previous SetRCBValues service request.

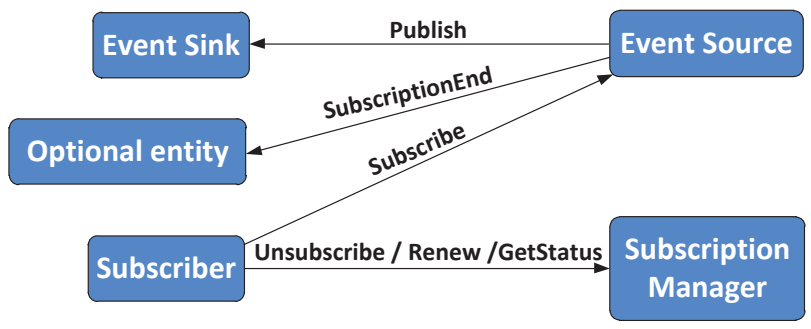

Fig. 3. WS-Eventing specification entities
With help of $W S_{\text {event }}$ mechanisms, OPC UA Notifications are published directly from Event source to Event sink without need of previous polling. Unsubscribe process has similar steps as the subscribe process except it forms adequate DeleteSubscription and DeleteMonitoredItems requests.

Described solution demonstrates unified approach for OPC UA and $W S_{\text {event }}$ notification capabilities. This mapping procedure is compliant with $a c s i_{r c b}$ functional requirements and compatible to application-logic of existing IEC 61850 equipment. Therefore, proposed solution can also be conveniently applied as a part of gateway for legacy IEC 61850 applications and devices.

Log-Control-Block (LCB) model $\left(a c s i_{l c b}\right)$ is used for retrieval of historical event related from device. This can be based on polling-based OPC UA event retrieval mechanism.

Combined usage of $u a_{\text {monit }}, u a_{\text {subs }}$ and $W S_{\text {event }}$ fulfills all functional requirements for vertical event-driven communication in IEC 61850 systems as shown in service alignment equations (4) and (5) and Fig. 4.

$$
\begin{aligned}
& a c s i_{r c b} \cong\left(u a_{\text {monit }} \cup u a_{\text {subs }} \cup W S_{\text {event }}\right) \\
& \operatorname{acsi}_{\text {lcb }} \cong\left(u a_{\text {monit }} \cup u a_{\text {subs }} \cup W S_{\text {event }}\right)
\end{aligned}
$$

As a conclusion it can be stated that functionalities required by $A C S I_{\text {vertical }}$ are represent subset of functionalities provided by $U A_{\text {services }}$ and $W S_{\text {event }}$ as show in in (6)-(8)

$$
\begin{gathered}
A C S I_{\text {vertical }}=\left\{\begin{array}{l}
a c s i_{\text {asso }}, a c s i_{\text {inform }}, a c s i_{\text {ctrl }}, \\
a c s i_{\text {sgcb }}, a c s i_{\text {rcb }}, a c s i_{\text {lcb }}
\end{array}\right\} \\
U A_{\text {services }}=\left\{\begin{array}{l}
u a_{\text {sess }}, u a_{\text {noma }}, u a_{\text {view }}, \\
u a_{\text {attr }}, u a_{\text {monit }}, u a_{\text {subs }}
\end{array}\right\} \\
A C S I_{\text {vertical }} \subseteq\left(U A_{\text {services }} \cup W S_{\text {event }}\right)
\end{gathered}
$$

\section{IEC 61850 Application \\ IEC 61850 Device}

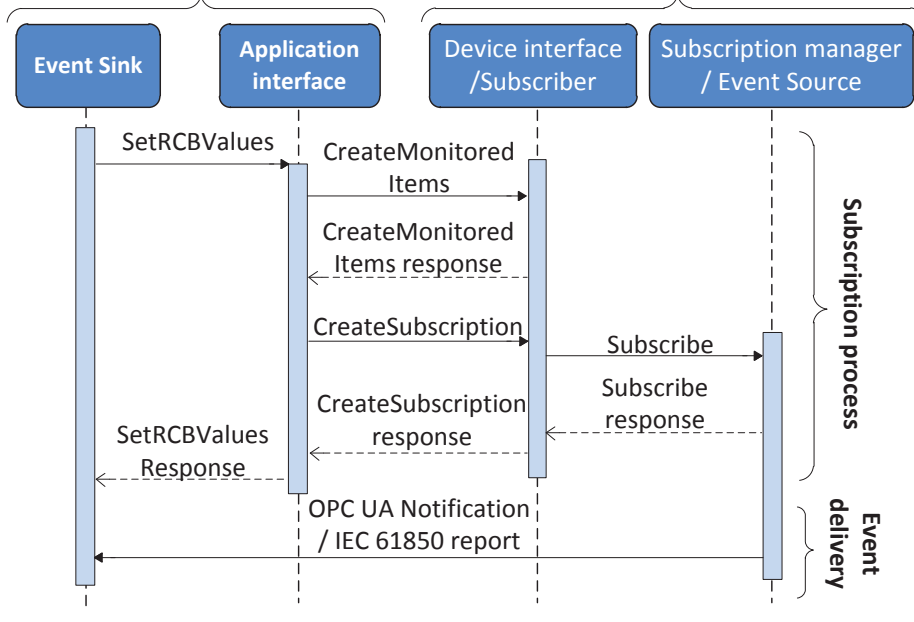

Fig. 4. Event-driven data exchange 


\section{CONCLUSION}

This article provides an analysis of application design requirements for novel Smart Grid domains such as DERs, EV supply stations, smart houses/buildings and identifies gaps in current IEC 61850 definition. In order to surpass these issues, a technical solution based on integrating devicelevel SOA design principles into IEC 61850 applications is proposed. Therefore, two most prominent industrial automation SOA technologies, DPWS and OPC UA, have been analyzed regarding Smart Grid integration requirements. As an analysis result, a new device-level SOA middleware platform for IEC 61850 is presented. A detailed analysis is provided for event-driven data exchange mechanism used in IEC 61850 systems. The proposed solution benefits both from DPWS and OPC UA and provides significant value-added features for accelerated Smart Grid deployment.

\section{ACKNOWLEDGMENT}

The authors would like to thank the European Commission for its support through the European Collaborative Research project IMC-AESOP of the FP7 program, which has partially funded this activity.

\section{REFERENCES}

[1] V. C. Gungor, D. Sahin, T. Kocak, S. Ergut, C. Buccella, C. Cecati, and G. P. Hancke, Smart Grid Technologies: Communication Technologies and Standards, IEEE Transactions on Industrial Informatics, vol. 7, no. 4, pp. 529-539, Nov. 2011.

[2] IEC, Communication Networks and Systems in Substations - ALL PARTS, Int. Std. IEC 61850-SER ed1.0, 2011.

[3] S. Sučić, T. Dragičević, T. Capuder, and M. Delimar, Economic dispatch of virtual power plants in an event-driven service-oriented framework using standards-based communications, Electric Power Systems Research, vol. 81, no. 12, pp. 2108-2119, 2011.

[4] W. Su, H. Rahimi Eichi, W. Zeng, and M. Chow, A Survey on the Electrification of Transportation in a Smart Grid Environment, IEEE Transactions on Industrial Informatics, vol. PP, no. 99, p. 1-1.

[5] F. Jammes and H. Smit, Service-oriented paradigms in industrial automation, IEEE Transactions on Industrial Informatics, vol. 1, no. 1, pp. $62-70$, Feb. 2005.

[6] A. P. Kalogeras, J. V. Gialelis, C. E. Alexakos, M. J. Georgoudakis, and S. A. Koubias, Vertical integration of enterprise industrial systems utilizing web services, IEEE Transactions on Industrial Informatics, vol. 2, no. 2, pp. 120-128, May 2006.

[7] G. Candido, A. W. Colombo, J. Barata, and F. Jammes, ServiceOriented Infrastructure to Support the Deployment of Evolvable Production Systems, IEEE Transactions on Industrial Informatics, vol. 7, no. 4, pp. 759-767, Nov. 2011.

[8] OASIS, 'Devices Profile for Web Services Version 1.1 Specification,' 2009, http://www.oasis-open.org/committees/ws-dd.

[9] OPC Foundation, 'OPC Unified Architecture (OPC-UA) Specifications,' 2011, http://www.opcfoundation.org/UA.

[10] A. Timbus, M. Larsson, and C. Yuen, Active Management of Distributed Energy Resources Using Standardized Communications and Modern Information Technologies, IEEE Transactions on Industrial Electronics, vol. 56, no. 10, pp. 4029-4037, Oct. 2009.

[11] M. Ruta, F. Scioscia, E. Di Sciascio, and G. Loseto, Semantic-Based Enhancement of ISO/IEC 14543-3 EIB/KNX Standard for Building Automation, IEEE Transactions on Industrial Informatics, vol. 7, no. 4, pp. 731-739, Nov. 2011.

[12] T. Sauter and M. Lobashov, End-to-End Communication Architecture for Smart Grids, IEEE Transactions on Industrial Electronics, vol. 58, no. 4, pp. 1218-1228, Apr. 2011.

[13] M. D. Galus, S. Koch, and G. Andersson, Provision of Load Frequency Control by PHEVs, Controllable Loads, and a
Cogeneration Unit, IEEE Transactions on Industrial Electronics, vol. 58, no. 10 , pp. 4568-4582, Oct. 2011.

[14] V. Vyatkin and G. Zhabelova, Multi-agent Smart Grid Automation Architecture based on IEC 61850/61499 Intelligent Logical Nodes, IEEE Transactions on Industrial Electronics, vol. PP, no. 99, p. 1-1.

[15] S. Sučić, H. Keserica, and D. Mileta, Information security standards for electrical power systems, in 32nd international convention on information and communication technology, electronics and microelectronics-MIPRO, 2009.

[16] IEC, Communication networks and systems for power utility automation - Part 7-2: Basic information and communication structure - Abstract communication service interface (ACSI), Int. Std. IEC 61850-7-2 ed2.0, 2010.

[17] T. Erl, Service-oriented architecture: concepts, technology, and design. Prentice Hall PTR, 2005.

[18] I. M. Delamer and J. L. Lastra, Service-Oriented Architecture for Distributed Publish/Subscribe Middleware in Electronics Production, IEEE Transactions on Industrial Informatics, vol. 2, no. 4, pp. 281294, Nov. 2006.

[19] B. Bony, M. Harnischfeger, and F. Jammes, Convergence of OPC UA and DPWS with a cross-domain data model, in 20119 th IEEE International Conference on Industrial Informatics (INDIN), 2011, pp. 187-192.

[20] G. Candido, F. Jammes, J. B. de Oliveira, and A. W. Colombo, SOA at device level in the industrial domain: Assessment of OPC UA and DPWS specifications, in 2010 8th IEEE International Conference on Industrial Informatics (INDIN), 2010, pp. 598-603.

[21] M. J. A. Izaguirre, A. Lobov, and J. L. Lastra, OPC-UA and DPWS interoperability for factory floor monitoring using complex event processing, in 2011 9th IEEE International Conference on Industrial Informatics (INDIN), 2011, pp. 205-211.

[22] S. Sučić, A. Martinić, and A. Kekelj, Utilizing standards-based semantic services for modeling novel Smart Grid supervision and remote control frameworks, in Industrial Technology (ICIT), 2012 IEEE International Conference on, 2012, pp. $409-414$.

[23] S. Sučić, A. Martinić, and D. Francesconi, Utilizing SOA-ready Devices for Virtual Power Plant Control in Semantic-enabled Smart Grid: Analyzing IEC 61850 and OPC UA integration methodology, in 2011 Second IEEE International Conference on Smart Grid Communications (SmartGridComm), 2011.

[24] S. Sučić, B. Bony, and Guise, L., Standards-compliant Event-driven SOA for Semantic-enabled Smart Grid Automation: Evaluating IEC 61850 and DPWS Integration, in Industrial Technology (ICIT), 2012 IEEE International Conference on, 2012, pp. 403 - 408.

[25] IEC, Communication networks and systems in substations - Part 8-1. Specific Communication Service Mapping (SCSM) - Mappings to MMS (ISO 9506-1 and ISO 9506-2) and to ISO/IEC 8802-3, IEC Std. IEC 61850-8-1 ed2.0, 2011.

[26] ISO, Industrial automation systems - Manufacturing Message Specification - ALL PARTS, Int. Std. ISO 9506 ed2.0, 2003.

[27] D. Booth, H. Haas, F. McCabe, E. Newcomer, M. Champion, C. Ferris, and D. Orchard, Web Services Architecture, W3C Working Group Note 11 February 2004, World Wide Web Consortium, article available from: http://www. w3. org/TR/ws-arch, p. 13, 2004.

[28] SOA4D - Service-oriented Architectures for Devices, Available: http://www.soa4d.org.

[29] WS4D - Web Services for Devices Initiative, Available: http://www.ws4d.org.

[30] IEC, Wind turbines Part - 25-4: Communications for monitoring and control of wind power plants - Mapping to communication profiles, Int. Std. IEC 61400-25-4 ed1.0, 2008.

[31] D. Griffin and D. Pesch, A Survey on Web Services in Telecommunications, IEEE Communications Magazine, vol. 45, no. 7, pp. 28-35, Jul. 2007. 